\title{
Giant reduction of InN surface electron accumulation: Compensation of surface donors by Mg dopants
}

\author{
W. M. Linhart, ${ }^{1}$ J. Chai, ${ }^{2, *}$ R. J. H. Morris,${ }^{1}$ M. G. Dowsett,${ }^{1}$ \\ C. F. McConville, ${ }^{1}$ S. M. Durbin, ${ }^{2,3,4}$ and T. D. Veal ${ }^{1,5, \dagger}$ \\ ${ }^{1}$ Department of Physics, University of Warwick, Coventry CV4 7AL, United Kingdom \\ ${ }^{2}$ Department of Electrical and Computer Engineering, \\ University of Canterbury, Christchurch, New Zealand \\ ${ }^{3}$ The MacDiarmid Institute for Advanced Materials and Nanotechnology, New Zealand \\ ${ }^{4}$ Department of Electrical Engineering and Department of Physics, \\ University at Buffalo, The State University of New York, Buffalo, New York, USA \\ ${ }^{5}$ Stephenson Institute for Renewable Energy and Department of Physics, \\ School of Physical Sciences, University of Liverpool, Liverpool L69 4ZF, United Kingdom
}

\begin{abstract}
Extreme electron accumulation with sheet density greater than $10^{13} \mathrm{~cm}^{-2}$ is almost universally present at the surface of indium nitride $(\mathrm{InN})$. Here, x-ray photoemission spectroscopy and secondary ion mass spectrometry are used to show that the surface Fermi level decreases as the $\mathrm{Mg}$ concentration increases, with the sheet electron density falling to below $10^{8} \mathrm{~cm}^{-2}$. Surface spacecharge calculations indicate that the lowering of the surface Fermi level increases the density of unoccupied donor-type surface states and that these are largely compensated by Mg-acceptors in the near-surface hole depletion region rather than by accumulated electrons. This is a significant step towards the realization of InN-based optoelectronic devices.
\end{abstract}

Indium nitride (InN) and associated In-rich InGaN alloys have enormous potential for use in optoelectronic applications, such as high efficiency solar cells. However, these opportunities have yet to be exploited due to the difficulties achieving a $p$ - $n$ junction device in the presence of surface electron accumulation/inversion layers characterised by a sheet electron density in excess of $10^{13} \mathrm{~cm}^{-2}$ [1-4], with the exception of biasing via an electrolyte or ionic liquid [5]. This electron accumulation can be explained by the extremely low conduction band minimum at the $\Gamma$ point of the Brillouin zone. Consequently the charge neutrality level (CNL) is located particularly high in the conduction band and the surface Fermi level is pinned below the CNL, resulting in an electron accumulation layer. In the majority of semiconductors, the CNL lies in the band gap at the $\Gamma$ point, which leads to surface electron depletion for $n$-type doping. The electron accumulation has been observed at the surface of wurtzite Inand N-polar $c$-plane InN and non-polar $a$ - and $m$-plane $\mathrm{InN}$, as well as at the surface of zinc-blende $\operatorname{InN}(001)$ $[2,6]$. Based on first-principles calculations, Segev and Van de Walle [7] proposed no electron accumulation layer would form at the surface of $a$-plane $(11 \overline{2} 0)$ and $m$-plane (1100) InN in the absence of In adlayers. This lack of electron accumulation was reported on in-situ cleaved $a$-plane InN [8], but has not been observed on as-grown surfaces [2, 8-11]. However, recent reports suggest that the In adlayers at the surface of $\mathrm{N}$-polar $c$-plane $\mathrm{InN}$ can be avoided under certain growth conditions or can be removed by chemical treatment. Such In adlayer-free Npolar surfaces have been shown to have much reduced electron accumulation [12, 13]. Additionally, the presence of an almost negligible level of electron accumulation has been reported at the lateral nonpolar surfaces of some InN nanowires [14]. Interestingly, there have been no reports of the removal or significant reduction of the electron accumulation at the surface of In-polarity InN.

In this letter, we report significant lowering of the surface Fermi level in Mg-doped $\left(>1 \times 10^{19} \mathrm{~cm}^{-3}\right)$ $\operatorname{InN}(0001)$ thin films with increasing $\mathrm{Mg}$-doping, indicating a large reduction of the electron accumulation. Surface space-charge calculations are used to determine the surface sheet electron density and donor surface state density as a function of $\mathrm{Mg}$-doping level. As the $\mathrm{Mg}$ doping is increased, the donor surface state density increases, but it is increasingly compensated by near-surface Mg acceptors rather than by free electrons.

The Mg-doped In-polarity InN samples were grown by plasma-assisted molecular beam epitaxy (PA-MBE) with a range of $\mathrm{Mg}$ cell temperatures at the University of Canterbury, New Zealand. All 1200 nm thick films were grown on a $c$-plane sapphire substrate with a 3000 $4000 \mathrm{~nm}$ GaN template layer, provided by Lumilog. The $\mathrm{Mg}$ concentration was determined by secondary ion mass spectrometry (SIMS) with an Atomika 4500 SIMS instrument using a $500 \mathrm{eV} \mathrm{O}_{2}^{+}$ion beam in conjunction with an ion-implanted InN standard. Before loading into the $\mathrm{x}$-ray photoelectron spectroscopy (XPS) vacuum chamber, the samples were subjected to etching in a $10 \mathrm{~mol} / \mathrm{l}$ $\mathrm{HCl}$ solution for $60 \mathrm{~s}$ to reduce the oxide layers, followed by rinsing in deionized water and immediate drying in $\mathrm{N}_{2}$. High-resolution XPS measurements were performed at room temperature using a monochromated $\mathrm{Al} K_{\alpha}$ xray source and a Scienta ESCA300 spectrometer at the National Centre for Electron Spectroscopy and Surface Analysis, Daresbury Laboratory, UK. The Fermi level was calibrated from the Fermi edge of an ion-bombarded silver reference sample; details of the spectrometer are re- 
ported elsewhere [15]. Core-level XPS analysis indicated that, after the cleaning treatment, the In-O bonding component of the In $3 d$ spectra was entirely absent, but a submonolayer coverage of residual adventitious oxygen and carbon remained on the samples. The In $3 d: \mathrm{N} 1 s$ intensity ratio indicated the presence of In adlayers on all the InN samples investigated. While in situ low energy electron diffraction (LEED) was not available in the XPS system, similar sample preparation has previously resulted in a clear $(1 \times 1)$ LEED pattern, indicating a relatively well-ordered surface.

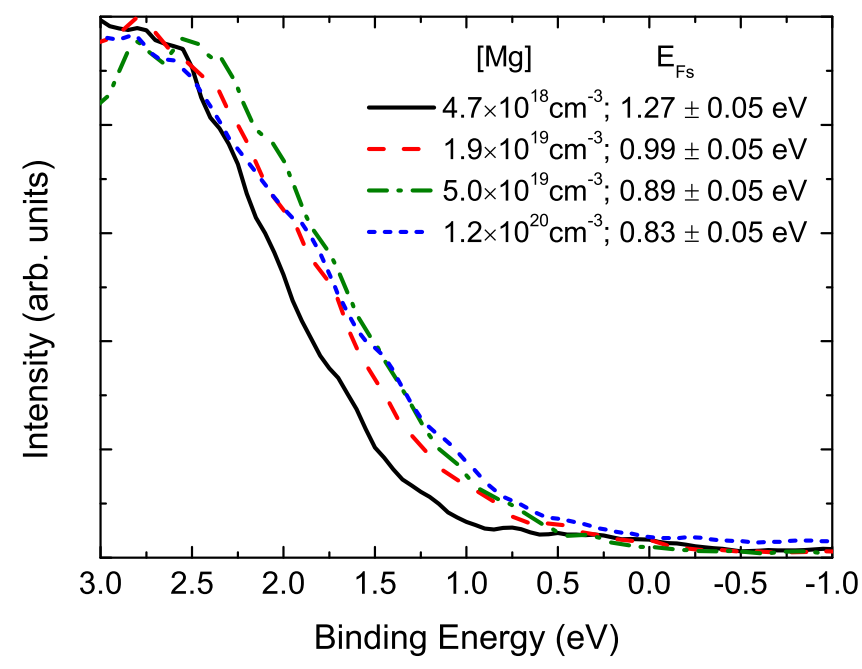

FIG. 1: (Color online) Valence band photoemission of the $\mathrm{Mg}$-doped $\mathrm{InN}$ for different $\mathrm{Mg}$ concentrations. The position of the surface Fermi level with respect to the valence band extracted from the measurement is quoted for each sample.

Valence band (VB) edge XPS spectra for high Mgdoped $\mathrm{InN}$ samples are shown in Fig. 1. The position of the surface Fermi level $\left(E_{F s}\right)$ with respect to the valence band maximum (VBM) was determined by linearly extrapolating the valence band leading edge to the intersection with the background level to account for the finite resolution of the spectrometer [16]. The surface Fermi levels derived from the valence band XPS data are plotted in Fig. 2 as a function of carrier concentration, where for the four most heavily Mg-doped samples, it is assumed that all the $\mathrm{Mg}$ detected by SIMS acts as an acceptor. This assumption is supported by our own electrochemical capacitance-voltage measurements of these samples, where the slope of the Mott-Schottky curves changes from positive to negative for the heavily $\mathrm{Mg}$ doped samples (not shown here), indicating that $\mathrm{InN}$ is p-type beneath the surface electron-rich layer [17-20].

For moderate $\mathrm{Mg}$ concentrations, $E_{F s}$ lies $1.3-1.4 \mathrm{eV}$ above the VBM [21, 22], which is identical to what has been previously reported using the same analysis method for all $n$-type samples with $n<1 \times 10^{19} \mathrm{~cm}^{-3}$ (Fig. 2). However, for the highest $\mathrm{Mg}$ concentrations $\left(>1 \times 10^{19} \mathrm{~cm}^{-3}\right)$, a significant lowering of $E_{F s}$ with in-

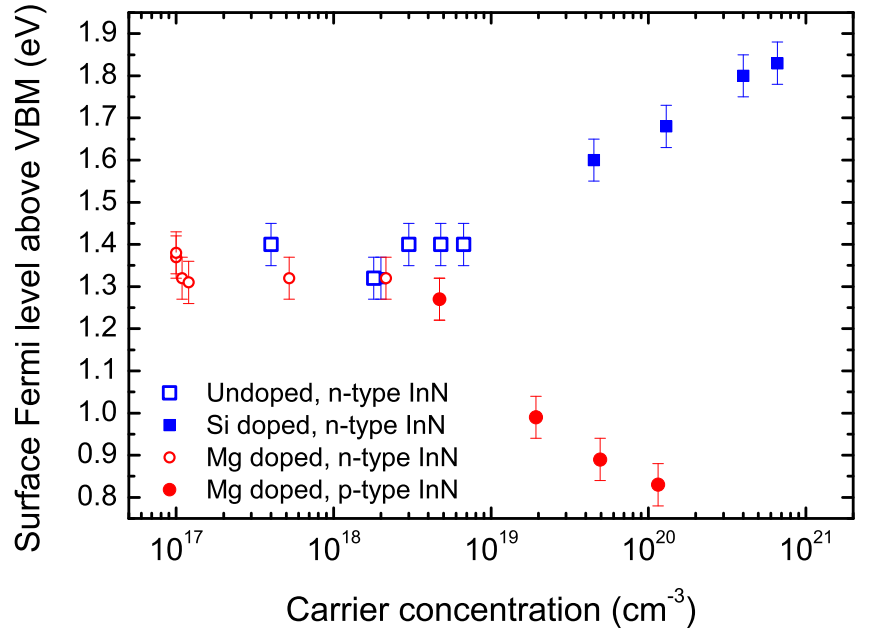

FIG. 2: (Color online) The surface Fermi level position for undoped n-type $\mathrm{InN}$ (open squares), Si-doped n-type $\mathrm{InN}$ (closed squares), n-type Mg-doped InN as Mg concentration too low to give p-type conductivity (open circles) and p-type Mg-doped InN (closed circles) as a function of carrier concentration. The electron concentration values of Si-doped InN were taken from ref. [26], whereas the hole concentrations in $\mathrm{Mg}$-doped $\mathrm{InN}$ were assumed to be equal to the $\mathrm{Mg}$ concentrations, in agreement with our ECV measurements and ref. [20].

creasing $\mathrm{Mg}$ concentration is observed. For $[\mathrm{Mg}]=1.2 \times$ $10^{20} \mathrm{~cm}^{-3}$, a value of $0.83 \mathrm{eV}$ above the VBM is found. Given a band gap of $0.64 \mathrm{eV}$ [23], the surface Fermi level is located above the bottom of the conduction band and above the bulk Fermi level for all the Mg-doped InN samples, indicating downward band bending at the surface in each case. The surface sheet electron density associated with the occupied In $5 s$-character conduction band is too low to be observed with $\mathrm{Al} \mathrm{K}_{\alpha}$ photoemission, but has been observed for $n$-type InN using synchrotron radiation photoemission with $7590 \mathrm{eV}$ photons for which the In $5 s: \mathrm{N} 2 p$ cross section ratio is greater [24].

The band bending and carrier concentration profiles as a function of depth from the surface can be calculated by numerically solving the Poisson equation within the modified Thomas-Fermi approximation (MTFA) [3] as shown in Fig. 3. Each profile was calculated using an inversion layer model, assuming that all $\mathrm{Mg}$ atoms act as acceptors and using a hole effective mass of $0.64 m_{e}[25]$. From Fig. 3, it can be seen that the electron accumulation near the surface and associated band bending are decreased with increasing $\mathrm{Mg}$ concentration. The ionized donor surface state density can be obtained from the gradient of the band bending potential at the surface,

$$
n_{s s}=\left.\frac{\varepsilon(0) \varepsilon_{0}}{e} \frac{d V}{d z}\right|_{z=0},
$$

and the surface sheet electron density, $n_{2 D}$, is found 

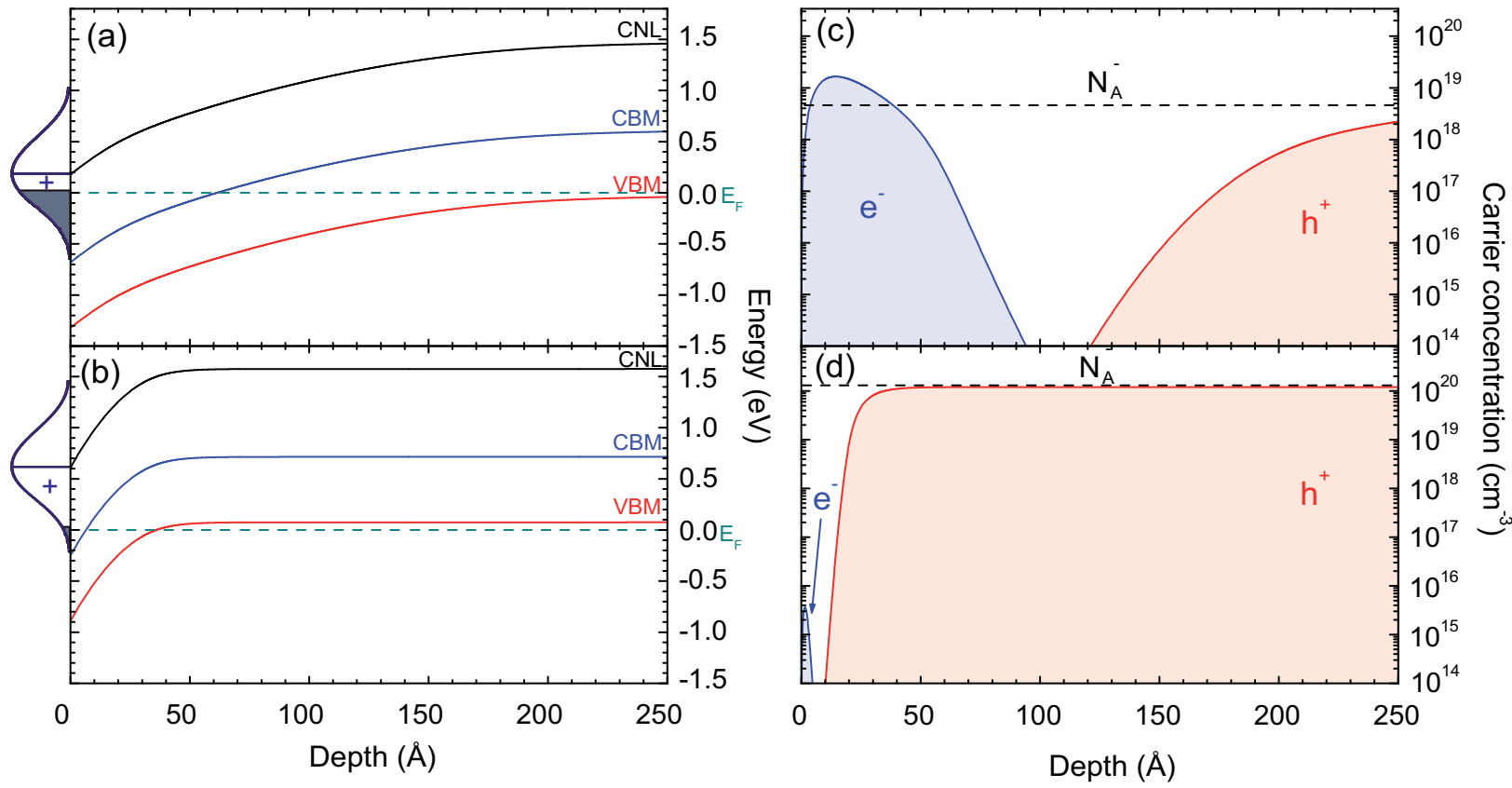

FIG. 3: (Color online) The conduction band minimum (CBM), valence band maximum and charge neutrality level (CNL) with respect to the Fermi level (dashed line), as a function of depth from the surface, for a $\mathrm{Mg}$ concentration of $(\mathrm{a}) 4.7 \times 10^{18} \mathrm{~cm}^{-3}$ and (b) $1.2 \times 10^{20} \mathrm{~cm}^{-3}$. The CNL is located high above the CBM [26]. The shaded portion in each case represents filled neutral donor states. The portion labelled "+" represents the ionized donor states. (c) and (d) shows the carrier concentration profiles corresponding to (a) and (b), respectively.

from the area under the electron accumulation peak in the carrier concentration profile. From the calculated charge-depth profiles, the surface sheet electron densities, $\mathrm{n}_{2 D}$, for the samples with the highest $\mathrm{Mg}$ concentration have been found to be between $7.9 \times 10^{7} \mathrm{~cm}^{-2}$ and $2.5 \times 10^{10} \mathrm{~cm}^{-2}$, several orders of magnitude lower than for undoped $\mathrm{InN}$ [22], while for the moderately $\mathrm{Mg}$ doped InN films, the $\mathrm{n}_{2 D}$ has been determined to be $\sim 1.0 \times 10^{13} \mathrm{~cm}^{-2}$. The $\mathrm{n}_{2 D}$ values as a function of $\mathrm{Mg}$ concentration are presented in Fig. 4 (a).

If the surface Fermi level, $E_{F s}$, is located below the charge neutrality level (CNL), some of the higher-energy donor-like surface states will be unoccupied and hence positively charged. Incorporation of $\mathrm{Mg}$ into InN shifts the Fermi level towards the VBM [17] and hence reduces the fraction of donor-like surface states which are occupied. Consequently, the positively charged surface donor density increases which might be expected to result in an increased electron accumulation layer; however, the surface electron sheet density (accumulation layer) decreases rather significantly (see Fig. 4) as the Mg concentration increases. The surface, in an equilibrium state, must be neutral; therefore the positive surface charge must be balanced. This can be understood within the inversion layer model. Instead of surface electrons compensating the positively charged surface donor states, as in the electron accumulation case for $n$-type $\operatorname{InN}$, this function is performed by the near-surface $\mathrm{Mg}$ acceptors, $\mathrm{N}_{A}^{-}$.
For the highest $\mathrm{Mg}$ concentration, the surface electron sheet density is dramatically reduced, therefore the sample essentially exhibits a hole depletion layer (Fig. 3 (b) and (d)). Similar compensation of bulk Zn acceptors by positively charged surface $\mathrm{P}$ vacancies has been reported for the $\operatorname{InP}(110)$ surface [28], but without the enormous change from the generally observed surface space-charge layer that is reported here. It should also be noted that XPS core level spectra did not show any signal from $\mathrm{Mg}$, indicating no significant $\mathrm{Mg}$ segregation to the surface of the films, the bulk doping concentration of $\mathrm{Mg}$ being too low for XPS to detect. This and the uniform $\mathrm{Mg}$ concentration seen in the SIMS profiles beneath the surface transient region support the use of a constant background acceptor density as a function of depth in the space-charge calculations.

As a result, for the case of $n$-type $\mathrm{InN}, n \sim 1 \times 10^{19}$ $\mathrm{cm}^{-3}$ is the doping level (see Fig. 2) above which the $E_{F s}$ of undoped and Si-doped InN tends to increase to reduce the electron accumulation, producing flat bands and maintaining charge neutrality [26]. In the case of $p$-type InN, the $E_{F s}$ level tends to decrease to reduce the electron accumulation, producing a hole depletion layer and maintaining charge neutrality. Similar divergence of the surface Fermi level position with doping was previously observed for $n$ - and $p$-type GaAs, the archetypal compound semiconductor [27]. However, rather than the surface states responsible for the space charge always be- 
ing donors as in the $\mathrm{InN}$ case, for GaAs, the type of charged surface state changes from acceptors in $n$-type to donors in $p$-type GaAs. Equivalently, downward band bending is observed for InN for both $n$ - and $p$-type doping, whereas upward band bending is present for $n$-type GaAs and downward for $p$-type. This difference is due to the respective positions of the CNL in these two materials, with that of InN lying above the conduction band minimum [26] and that of GaAs is just below the middle of the fundamental band gap [29, 30].
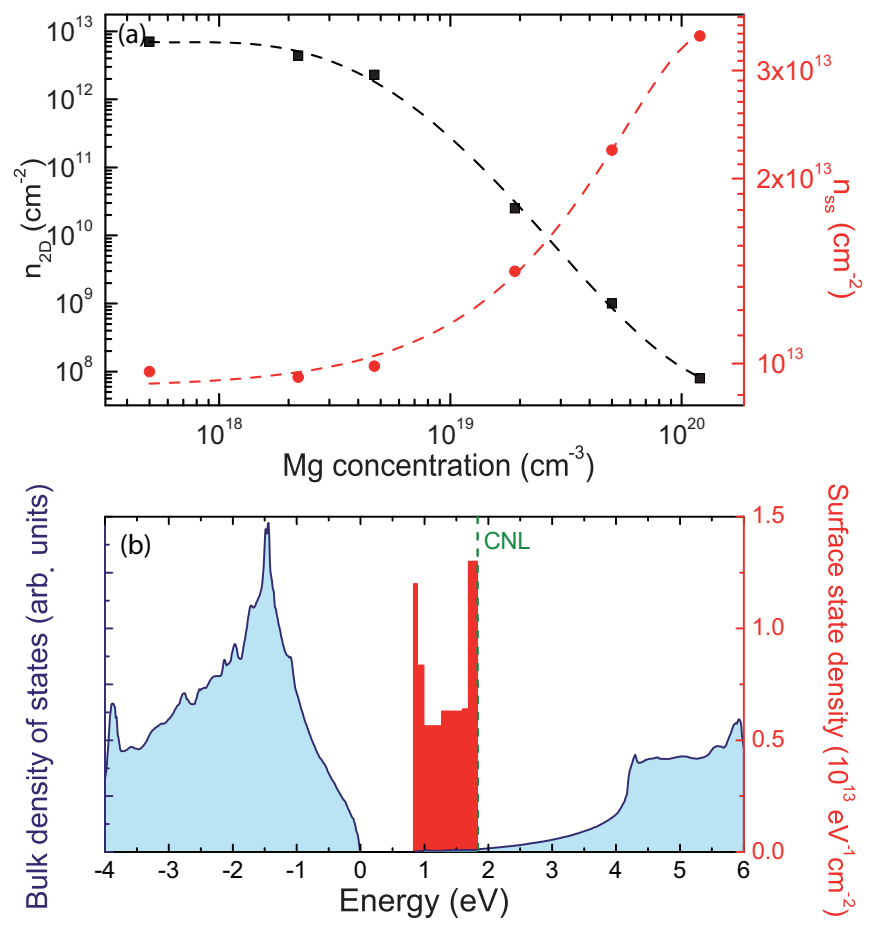

FIG. 4: (Color online) (a) Surface electron sheet density $\mathrm{n}_{2 D}$ and donor-like ionized surface state density $\mathrm{n}_{s s}$ as a function of $\mathrm{Mg}$ concentration. (b) Surface state density (red histogram) as a function of energy. The height of each bar in the histogram is evaluated by taking the difference in surface state density between two successive samples and the width is determined by the difference between their surface Fermi levels. The bulk density of states (blue line) for InN calculated by Furthmüller et al [31], using quasiparticle-corrected density-functional theory is also shown.

By combining XPS measurements and space charge calculations, it is possible to determine the density of surface states as a function of energy for comparison with the theoretical predictions of Segev and Van de Walle $[7,32,33]$. For each of the Mg-doped, undoped and Sidoped samples, the surface Fermi level was measured by XPS and the unoccupied donor surface state density derived by solving the Poisson equation. A histogram profile of the density of states was then created by taking the difference between the surface state densities for successive pairs of samples over the energy range defined by the difference between their surface Fermi levels. The re- sults are presented in Fig. 4(b) and plotted with the bulk density of states calculated using quasiparticle-corrected density-functional theory [31]. The lower energy peak is associated with the large differences in $n_{s s}$ at high $\mathrm{Mg}$ concentrations, that is, from the data points for $\mathrm{Mg}$ concentrations of greater than $1 \times 10^{19} \mathrm{~cm}^{-3}$. Similarly, the higher energy peak near the CNL is derived from the large differences in $n_{s s}$ for InN samples with high $\mathrm{Si}$ concentrations of greater than $1 \times 10^{19} \mathrm{~cm}^{-3}$ which are associated with the surface Fermi levels shown in Fig. 2 and band bending values reported in Ref. [26]. The two peak structure of the experimentally determined surface density of states is in qualitative agreement with the theoretical surface density of states which was associated with In-In bonding in an In adlayer at the $\operatorname{InN}(0001)$ surface by Segev and Van de Walle [7, 32, 33].

In conclusion, we have demonstrated that incorporation of $\mathrm{Mg}$ as a dopant into $\mathrm{InN}$ redefines the bulk and surface electronic properties of this material. Previous results have shown that $\mathrm{Si}$ acts as a donor and its incorporation increases the bulk electron concentration. For $n>1 \times 10^{19} \mathrm{~cm}^{-3}$, the donor-like surface state density decreases and $E_{F s}$ saturates at the CNL, indicating a transition from an accumulation layer to flat bands. In contrast, $\mathrm{Mg}$ acts as an acceptor and its presence changes the type of conductivity from $n$-type to $p$-type. For $[M g]>1 \times 10^{19} \mathrm{~cm}^{-3}$, the donor surface state density increases while $E_{F s}$ decreases; however, the surface sheet electron density decreases enormously, indicating a transition from electron accumulation to hole depletion for heavily $\mathrm{Mg}$-doped $\mathrm{InN}$. As the $\mathrm{Mg}$ concentration increases, the donor surface states are increasingly compensated by Mg near-surface acceptors in the hole depletion region. These results give positive indications for fabrication of InN $p$ - $n$ junctions, because electron accumulation naturally disappears at high $\mathrm{Mg}$ doping levels.

The authors are grateful to F. Bechstedt for valuable discussions. We acknowledge support from RAINBOW, a Marie Curie Initial Training Network funded by the European Commission under the 7th Framework programme, Grant agreement $N^{\circ}$ :PITN-GA-2008-213238, the Engineering and Physical Sciences Research Council, UK, under Grant No. EP/G004447/1, the MacDiarmid Institute for Advanced Materials and Nanotechnology, and the State University of New York.

* Current address: Queensland Micro- and Nanotechnology Centre, Griffith University, Nathan 4111, Australia.

$\dagger$ Electronic address: T.Veal@liverpool.ac.uk

[1] I. Mahboob, T. D. Veal, C. F. McConville, H. Lu, and W. J. Schaff, Phys. Rev. Lett. 92, 036804 (2004).

[2] P. D. C. King, T. D. Veal, C. F. McConville, F. Fuchs, J. Furthmüller, F. Bechstedt, P. Schley, R. Goldhahn, J. Schörmann, D. J. As, K. Lischka, D. Muto, H. Naoi, 
Y. Nanishi, H. Lu, and W. J. Schaff, Appl. Phys. Lett. 91, 092101 (2007)

[3] T. D. Veal, L. F. J. Piper, W. J. Schaff, and C. F. McConville, J. Cryst. Growth 288, 268 (2006).

[4] R. E. Jones, K. M. Yu, S. X. Li, W. Walukiewicz, J. W. Ager, E. E. Haller, H. Lu, and W. J. Schaff, Phys. Rev. Lett. 96, 125505 (2006).

[5] E. Alarcón-Lladó, M. A. Mayer, B. W. Boudouris, R. A. Segalman, N. Miller, T. Yamaguchi, K. Wang, Y. Nanishi, E. E. Haller, and J. W. Ager, Appl. Phys. Lett. 99, 102106 (2011)

[6] W. M. Linhart, T. D. Veal, P. D. C. King, G. Koblmüller, C. S. Gallinat, J. S. Speck, and C. F. McConville, Appl. Phys. Lett. 97, 112103 (2010).

[7] D. Segev and C. G. Van de Walle, Europhys. Lett 76, 305 (2006).

[8] C.-L. Wu, H.-M. Lee, C.-T. Kuo, C.-H. Chen, and S. Gwo, Phys. Rev. Lett. 101, 106803 (2008).

[9] H. Lu, W. J. Schaff, L. F. Eastman, J. Wu, W. Walukiewicz, V. Cimalla, and O. Ambacher, Appl. Phys. Lett. 83, 1136 (2003).

[10] E. Calleja, J. Grandal, M. A. Sánchez-García, M. Niebelschütz, V. Cimalla, and O. Ambacher, Appl. Phys. Lett. 90, 262110 (2007).

[11] T. Nagata, G. Koblmüller, O. Bierwagen, C. S. Gallinat, and J. S. Speck, Appl. Phys. Lett. 95, 132104 (2009).

[12] C.-T. Kuo, S.-C. Lin, K.-K. Chang, H.-W. Shiu, L.-Y. Chang, C.-H. Chen, S.-J. Tang, and S. Gwo, Appl. Phys. Lett 98, 052101 (2011)

[13] A. Eisenhardt, M. Himmerlich, and S. Krischok, Phys. Stat. Sol. A 209, 45 (2012).

[14] Y.-L. Chang, Z. Mi, and F. Li, Adv. Funct. Mater. 20, 4145 (2010)

[15] P. D. C. King, T. D. Veal, P. H. Jefferson, C. F. McConville, H. Lu, and W. J. Schaff, Phys. Rev. B 75, 115312 (2007)

[16] S. A. Chambers, T. Droubay, T. C. Kaspar, and M. Gutowski, J. Vac. Sci. Technol. B 22, 2205 (2004).

[17] P. A. Anderson, C. H. Swartz, D. Carder, R. J. Reeves, S. M. Durbin, S. Chandril, and T. H. Myers, Appl. Phys. Lett. 89, 184104 (2006).

[18] N. Miller, J. W. Ager III, H. M. Smith III, M. A. Mayer, K. M. Yu, E. E. Haller, W. Walukiewicz, W. J. Schaff,
C. Gallinat, G. Koblmüller, et al., J. Appl. Phys. 107, $113712(2010)$

[19] J. W. L. Yim, R. E. Jones, K. M. Yu, J. W. Ager III, W. Walukiewicz, W. J. Schaff, and J. Wu, Phys. Rev. B 76 041303(R) (2007).

[20] X. Wang, S.-B. Che, Y. Ishitani, and A. Yoshikawa, Appl. Phys. Lett. 91, 242111 (2007).

[21] T. D. Veal, P. D. C. King, P. H. Jefferson, L. F. J. Piper, C. F. McConville, H. Lu, W. J. Schaff, P. A. Anderson, S. M. Durbin, D. Muto, et al., Phys. Rev. B 76, 075313 (2007).

[22] P. D. C. King, T. D. Veal, C. S. Gallinat, G. Koblmüller, L. R. Bailey, J. S. Speck, and C. F. McConville, J. Appl. Phys. 104, 103703 (2008).

[23] J. Wu, W. Walukiewicz, W. Shan, K. M. Yu, J. W Ager III, S. X. Li, E. E. Haller, H. Lu, and W. J. Schaff, J. Appl. Phys. 94, 4457 (2003).

[24] T. D. Veal, P. D. C. King, D. Payne, C. F. McConville, G. Pannaccione, P. Lacovig, S. M. Durbin, and R. G. Egdell, unpublished.

[25] K. Wang, N. Miller, R. Iwamoto, T. Yamaguchi, M. A. Mayer, T. Araki, Y. Nanishi, K. M. Yu, E. E. Haller, W. Walukiewicz, et al., Appl. Phys. Lett. 98, 042104 (2011).

[26] P. D. C. King, T. D. Veal, P. H. Jefferson, S. A Hatfield, L. F. J. Piper, C. F. McConville, F. Fuchs, J. Furthmüller, F. Bechstedt, H. Lu, et al., Phys. Rev. B 77, 045316 (2008).

[27] M. D. Pashley, K. W. Haberern, R. M. Feenstra, and P. D. Kirchner, Phys. Rev. B 48, 4612 (1993).

[28] Ph. Ebert, M. Heinrich, M. Simon, C. Domke, K. Urban, C. K. Shih, M. B. Webb, and M. G. Lagally, Phys. Rev. B 53, 4580 (1996).

[29] J. Tersoff, Phys. Rev. B 32, 6968 (1985).

[30] W. Mönch, Appl. Surf. Sci. 117, 380 (1997).

[31] J. Furthmüller, F. Fuchs, F. Bechstedt in T. D. Veal, C. F. McConville and W. J. Shaff (Editors), Indium Nitride and Related Alloys (CRC Press, 2009), page 292.

[32] C. G. Van de Walle and D. Segev, J. Appl. Phys. 101, 081704 (2007)

[33] D. Segev and C. G. Van de Walle, J. Cryst. Growth 300 199 (2007) 